\section{Fine Structure in the Arc Spectra of Bromine} and Iodine.

Bromine.-The fine structures of a number of bromine arc lines have been previously reported by Hori. ${ }^{1}$ By assuming that the lines $\lambda \lambda 6632,6560,6351$, 6149 have identical structures, De Bruin ${ }^{2}$ inferred that the nuclear spin is $\frac{3}{2}$. The fine structure measurements have been considerably extended, using a high frequency (15 megacycles) electrodeless discharge in pure bromine vapour and a Fabry-Perot interferometer. All the observed structures arise from the $4 p^{4} 5 s$ electron configuration, as was to be expected. Although the lines employed by $\mathrm{De}$ Bruin have not identical structures, as he supposed, the value of $i=\frac{3}{2}$ has been confirmed. There is evidence that the two isotopes of bromine $(79,81)$ have the same nuclear spin.

Iodine.-Fine structures have been previously recorded only in the iodine spark lines, ${ }^{3}$ but by employ ing similar experimental arrangements to those used for bromine, fine structures have been observed in the region $\lambda 4700-\lambda 8000$. The are lines are mostly regular quartets and sextets degrading to the violet in both intensity and interval. The simple regularity of the structures is such that they are obviously characteristic of only one $j$ term in each line. The observed structures arise from the $5 p^{4} 6 s$ electron configuration, that is, that corresponding to the $4 p^{4} 5 s$ in bromine. This is in agreement with the partial analysis of the spectrum made here by S. F. Evans (unpublished). As $2 i+1$ is the maximum multiplicity attainable, the existence of sextet terms proves that $i$ is at least equal to $\frac{5}{2}$, which had been previously inferred from the absence of appreciable alternating intensities in the absorption band lines of $\mathrm{I}_{2}$.

Since with $j$ less than $i$ the full multiplicity is not attained, the application of the interval rule must decide the value of $i$ (in the absence of Zeeman effect measurements). The best line observed is $\lambda 4862$, which is a quartet and thus involves $j=\frac{3}{2}$ in the $5 p^{4} 6 s$ term (this is confirmed by the analysis). The intervals are $123,103,83\left(\mathrm{~cm}^{-1} \times 10^{-3}\right)$, that is, $6 \times 20.5,5 \times 20.6,4 \times 20.7$. A value of $i=\frac{3}{8}$ must be invoked to fit these exact ratios $(6: 5: 4)$, and this value is supported by the interval ratios in other lines.

The most probable values of $i$ for the halogens are shown in the table below :

\begin{tabular}{|l|c|c|c|c|}
\hline Halogen . . & Fluorine. & Chlorine. & Bromine. & Iodine. \\
\hline Number of Protons & 19 & $35(37,39)$ & 79,81 & 127 \\
\hline Nuclear Spin . & $\frac{1}{2}$ & $\frac{5}{2}$ & $\frac{3}{2}$ & $\frac{9}{2}$ \\
\hline
\end{tabular}

No apparent regularity exists, but the large and small $i$ values are associated with a single isotope. Full details of the fine structure measurements will be published elsewhere.

\section{Physics Department, Armstrong College (Newcastle-on-Tyne), Durham University, May 5. \\ 1 Hori, Mem. Coll. Sci. Kyoto, vol. 9, p. 307; 1926 \\ 2 De Bruin, Nature, Mar. 15, 1930, vol. 125, p. 414 \\ 3 Wood and Kimura, Astroph. Jour., vol, 46, p. 181; 1917.}

The Resonance Potential of Trebly Ionised Bismuth.

UsING the known data of the spectra $\mathrm{Hg} \mathrm{I,1} \mathrm{Tl}$ II, ${ }^{2}$ and $\mathrm{Pb}$ III, ${ }^{3}$ and extrapolating by means of the irregular doublet law, the predicted values of the wave numbers of the important combinations $6 s 6 s{ }^{1} S_{0}-6 s 6 p{ }^{3} P_{1}$ and $6 s 6 s{ }^{1} S_{0}-6 s 6 p{ }^{1} \mathrm{P}_{1}$ of $\mathrm{Bi}$ IV are approximately 76,000 and 115,000 .

No. 3214, VoL. 127]
In a list of wave-length measurements of the vacuum spark of bismuth in the Schumann region, for which I am indebted to Dr. R. J. Lang, there are very strong lines at $75923(\lambda 1317 \cdot 12 \mathrm{~A}$.$) and 114601$ $(\lambda 872.59 \mathrm{~A}$. $)$. If these are assumed to be the abovementioned combinations, then $6 s 6 p{ }^{3} P_{1}-6 s 6 p{ }^{1} P_{1}$ would be 38678 . A distinctive feature of the spectra $\mathrm{Hg} \mathrm{I}, \mathrm{Tl}$ II, and $\mathrm{Pb}$ III is the appearance with great intensity of lines arising from intercombinations between singlet and triplet terms. One would therefore expect to find the wave number difference 38678 recurring a number of times in the Schumann region. At least five pairs of lines with this difference have been found in approximately the expected positions. However, in spite of this apparent corroboration, I have been somewhat doubtful of the validity of the foregoing identification, because the line 75923 had already been included by Lang * in a scheme for $\mathrm{Bi}$ III. In a recent paper ${ }^{5}$ by McLennan, McLay, and Crawford on the spark spectra of bismuth, the line 75923 finds no place in the scheme for Bi II or for Bi III.

Still more significant evidence that this line belongs to $\mathrm{Bi} I V$, and is in fact the resonance line $6 s 6 s^{1} S_{0}-$ $6 s 6 p{ }^{3} P_{1}$, is to be found in the data given by Arvidsson $^{6}$ in a recent letter to NATURE, in which it is reported that the line 75923 has been resolved into three components of relative intensities $6: 5: 5$. If it is assumed that the nuclear quantum number of bismuth is $9 / 2$ and that the $6 s 6 s^{1} S_{0}$ term is single, then the theoretical relative intensities of the three components of the $6 s 6 s^{1} S_{0}-6 s 6 p{ }^{3} P_{1}$ combination as calculated by the formulæ given by Pauling and Goudsmit $^{7}$ is $6: 5: 4$ in order of decreasing wave number. This agrees very closely with the experimental ratio. It therefore seems very probable that this is the resonance line of $\mathrm{Bi} I V$, giving the value $9 \cdot 36$ volts for the resonance potential.

Some progress has been made in finding further wave number regularities in $\mathrm{Bi}$ IV, but the spectrum is difficult to interpret because of the $j j$ coupling of the two electrons. The work is still proceeding and it is hoped that a detailed report will be made elsewhere at a later date.

University of Alberta,

Stanley Smith. Edmonton, Canada, April 10.

Fowler, A., "Series in tine Spectra ", p. 148 ; Fleetway Press (1922), 2 McLennan, J. C. McLay, A. B., Crawford, M. F., Trans. Roy. Soc. Canada, 22, p. 241; 1928 . Smith, S., Proc. Nat. Acad. Sci., 14, p. 951; 1928.

${ }^{3}$ Rao, K. R., Narayan, A. L., Rao, A. S., Indian Jour, Phys., 2, p. 467 ; 1928. Smith, S., Proc. Nat. Acad. Sci., 14, p. 878; 1928

Lang, R. J. Phys Rev,

5 Mclennan, J. C., McLay, A. B., Crawford, M. F., Proc. Roy. Soc., $A, 129$, p. $579 ; 1930$

Arvidsson, G., NATURE, 126, p. 566 ; 1930.

" Pauling, L., and Goudsmit, S., " Structure of Line Spectra ", P. 140 and p. 214; MeGraw Hill (1930).

\section{Impact Figures on Polished Rock Salt Surfaces.}

IF a small steel ball is dropped from a height of a few inches on a polished rock salt surface, the imprint of the ball on the crystal surface remains as a circular depression of one or two millimetres in diameter. The surface is deformed, however, over a region many times the area of the circular depression. If an optical test plane (a piece of ordinary plate-glass will do) is placed on the crystal and the surface examined in monochromatic light, the interference pattern shows a number of families of 'loops' extending away from the imprint of the ball.

If the crystal surface approximates a $1,0,0$ plane, the depression will be surrounded by eight sets of 